A manuscript prepared for Vibrational Spectroscopy

\title{
Selection of Modulation Frequency of FT-IR Equipped with an MCT Detector for Thin-Film Analysis
}

Takeshi Hasegawa $^{* 1}$, Katsuhiko Taniguchi ${ }^{1}$ and Yoshiko Sato ${ }^{2}$

${ }^{1}$ Department of Chemistry, School of Engineering, Tokyo Institute of Technology, 2-12-1

Ookayama, Meguro-ku, Tokyo 152-8551, Japan

${ }^{2}$ Applied Molecular Chemistry, College of Industrial Technology, Nihon University, 1-2-1

Izumi-cho, Narashino, Chiba 275-8575, Japan

A revised manuscript of VIBSPEC-D-08-00154

*To whom correspondence should be addressed.

E-mail: hasegawa@chem.titech.ac.jp

${ }^{2}$ The present address (Yoshiko Sato): 1st Section, 1st Technical Department, 1st Production

Division, OptoMaterials Operations, Dai Nippon Printing Co., Ltd, 642-8 Mitsu-Ugaki, Okayama 709-2121, Japan 


\begin{abstract}
:
Optimal modulation frequency (scan rate) of an FT-IR equipped with a mercury-cadmium-telluride (MCT) detector has been investigated for obtaining high quality spectra of a monolayer-level thin film. Since an MCT detector is a semiconductor light device, it does not respond to modulation frequency significantly in comparison to a pyroelectric detector. A very thin film, however, requires high-throughput measurements to have high signal-to-noise ratio. In the present study, a balance of throughput and measurement time has been investigated by varying modulation frequency by using two spectrometers that have different sampling frequencies. In the present study, the stability of the baseline of absorbance spectra was also investigated depending on modulation frequencies, which suggested that $60 \mathrm{kHz}$ was most suitable for quantitatively reliable transmission measurements of Langmuir-Blodgett films.
\end{abstract}

Key words: FT-IR; Modulation frequency; MCT detector; Thin film 
Introduction: Fourier transform infrared (FT-IR) spectrometer has greatly been improved in the past two decades, and organic ultrathin films represented by Langmuir-Blodgett films ${ }^{1}$ even at a single-monolayer level have become a suitable analyte for FT-IR. The significant progress of FT-IR was realized mainly by technology progress in electronics about a mercury-cadmium-telluride (MCT) detector ${ }^{2}$ and the dynamic alignment of interferometer. ${ }^{2}$ In early 1990s, measurements of a single-monolayer LB film were a challenging task ${ }^{3,4}$ even with a high-sensitive optical geometry such as reflection-absorption spectrometry. ${ }^{5-7}$ Multi-layered LB films were therefore fabricated to overcome the analytical limitation. Thanks to the advances in technology, the situation was largely improved, and now a single-monolayer LB film can be measured even with transmission spectrometry.

An MCT detector is a semiconductor light device operated at a liquid-nitrogen cooled temperature, which has characteristics that it significantly responds to weak-intensity light while it does not greatly respond to modulation frequency (scanning rate or mirror velocity) in comparison to a pyroelectric detector. ${ }^{2}$ The lowest detectivity limit of a MCT detector can be about ten to fifty times greater than a pyroelectric detector such as deuterated triglycine sulfate (DTGS) detector. ${ }^{2}$ Therefore, when an MCT detector is coupled with a high-bit analogue-to-digital converter and amplifier, high-resolution measurements of light 'intensity’ can be performed, which leads to 'high sensitivity in absorbance.' An MCT detector is thus often used 
for measurements of infrared spectra of ultrathin films.

Another important improvement is found in the technology of dynamic alignment, ${ }^{2}$ which proves the stability of interferometer operation. Highly accurate operation of the interferometer in an FT-IR is definitely necessary for quantitatively reliable measurements of interferograms. Due to the development of dynamic alignment by computer-aided mechatronics, the operation has come close to ideal, which made accurate accumulation measurements possible.

In the present study, we have found that even a state-of-the-art spectrometer exhibits a theoretically unexpected response to modulation frequency. We have investigated responses of two spectrometers in our laboratory, one of which was assembled about fifteen years ago, while the other was made very recently. The old and new spectrometers employ different sampling frequencies of 100 and $500 \mathrm{kHz}$, respectively, but both are enough above the Nyquist frequency even when mid-infrared measurements at $4000 \mathrm{~cm}^{-1}$ or lower and the highest modulation frequency $(200 \mathrm{kHz})$ are taken into account. The Nyquist frequency, $f_{\text {samp }}$, in FT-IR is defined as:

$$
f_{\text {samp }}=2 V \tilde{v}_{\max }
$$

Here, $V$ is the optical velocity, and $\tilde{v}_{\max }$ is the highest wavenumber for the measurement. For a condition the highest modulation frequency on a NIR measurement at $12000 \mathrm{~cm}-1$, for example, the Nyquist frequency is calculated to be $304 \mathrm{kHz}$. Therefore, if we want to measure NIR spectra 
with a very rapid scanning rate, the new spectrometer should be chosen. For measurements of ordinary mid-infrared spectra, however, both spectrometers are considered to have no problem as long as only the sampling frequencies are taken into account. In theory, therefore, a very fast scanning can be chosen for mid-infrared measurements, which is expected to play a good role in a long time measurement.

When a very high modulation frequency was chosen, nevertheless, the measurement time has been found to be out of theoretical values largely, and this trend was very common to both the spectrometers. Then, an optimized experimental condition for measurements of ultrathin films has been explored. The modulation frequency was studied with respect to optical throughput, efficiency of accumulations and quantitative stability of absorbance spectra. As a result, the optimization was found to be meaningful to use an FT-IR with high reliability for better analysis of ultrathin films.

Experimental: The infrared spectrometers used for the present study were Thermo Fisher Scientific (Madison, WI) Magna 550 FT-IR and Nicolet 6700 both equipped with an MCT/A detector of an identical model that covers a mid-infrared range of $650-4000 \mathrm{~cm}^{-1}$. Measurements were performed after attaining a thermally stable condition of the detector, which was at least two hours after filling the vacuum bottle on the detector with liquid nitrogen. To make the experimental condition the same as that used for our practical analysis of thin films, 
2000 double-sided interferogram patterns were co-added for each measurement. The resolution was $4 \mathrm{~cm}^{-1}$.

The FT-IR has a variation of mirror velocity ( v): 0.1581, 0.3165, 0.4747, 0.6329, 0.9494, 1.2659, 1.8988, 2.5317, 3.1647, 3.7974, 4.4303, 4.7468, 5.0632, 5.6961 and $6.3290 \mathrm{~cm} \mathrm{sec}^{-1}$, which correspond to modulation frequencies $(f)$ of $5,10,15,20,30,40,60,80,100,120,140$, 150, 160, 180 and $200 \mathrm{kHz}$ (refer to Eq. 1), respectively, since a He-Ne laser with the wavelength $(\lambda=1 / \tilde{v})$ of $632 \mathrm{~nm}$ is equipped in the interferometer. ${ }^{8}$ The default "settle time" of $200 \mathrm{~ms}$ was used for the present study. The settle time is an implicit parameter controlling a stepping time before the next retardation of the moving mirror.

In the present paper, 'modulation frequency' is used instead of 'mirror velocity' unless otherwise stated, since mirror velocity is often confusing with 'optical path difference (OPD) velocity' or 'optical velocity,' which is defined considering the traveling distance of light between the fixed and moving mirrors.

The standard sample for the absorbance spectra measurements was a five-monolayer LB film of cadmium stearate deposited on a germanium substrate. The refractive index of germanium is extraordinary high, which makes observed band intensities (absorbance) smaller than using a low-refractive-index substrate such as $\mathrm{CaF}_{2}$. Regardless, the germanium substrate is often employed for our purposes, since it is chemically stable especially in an aqueous solution in 
comparison to materials of ionic salt, which is suitable for fabrication of LB films. Another reason to choose the germanium substrate is that the MAIRS technique requires a high refractive-index substrate. ${ }^{8-10}$

The LB deposition was performed at a surface pressure of $30 \mathrm{mN} \mathrm{m}^{-1}$ on a subphase surface. The water subphase for the LB deposition was comprised of $3 \times 10^{-4} \mathrm{M}$ cadmium chloride and $3 \times 10^{-4} \mathrm{M}$ sodium hydrocarbonate, so that $\mathrm{pH}$ should be ca. 7.3 , with which the stearic acid monolayer would fully be reacted to be cadmium salt at the subphase surface. Water used for the subphase was prepared by purification of tap water by a Millipore (Molsheim, France) Elix UV-3 pure-water generator and a Yamato (Tokyo, Japan) Autopure WT100U water purifier, which is a compatible model with Milli-Q. The resistivity of the product water was 18.3 $\mathrm{M} \Omega \mathrm{cm}$ and the surface tension was $72.8 \mathrm{mN} \mathrm{m}^{-1}$ at $25^{\circ} \mathrm{C}$, which proved that the water was contamination free.

Results and Discussion: Single-beam spectra of air (with no sample) were collected at various modulation frequencies in the range of $5-200 \mathrm{kHz}$. Intensity variations of the single-beam spectra measured at $2600 \mathrm{~cm}^{-1}$ for the sampling frequencies of 100 and $500 \mathrm{kHz}$ are plotted in Figure 1. Since the measurements were performed on the two spectrometers by using different built-in optical mesh filters to prevent saturation of the detector, the absolute intensities are different from each other. The entire variations are, however, found to be highly similar to each 
other even for a high modulation frequency region, although the two spectrometers have different sampling frequencies. It is commonly found that the variation exhibits almost no increase when the modulation frequency is less than $20 \mathrm{kHz}$, whereas it exhibits a linear increase by about $5 \%$ until the modulation frequency attains at $150 \mathrm{kHz}$. The sensitivity of a detector is evaluated by the use of 'specific detectivity', $D^{*}$, that is defined as: ${ }^{11}$

$$
D^{*}=\frac{\left(A_{D}\right)^{1 / 2}}{N E P}
$$

where $A_{D}$ is the detector area, and NEP (noise equivalent power) is a measure of the lowest limit of observable signal, which is comparable to the detector noise. NEP is defined as:

$$
\mathrm{NEP}=\frac{\mathrm{V}_{\mathrm{n}}}{\mathrm{R}_{\mathrm{V}}}
$$

where $V_{n}$ is the root mean square detector noise voltage, and $R_{v}$ is the voltage responsibility. NEP should be as small as possible to measure low intensity radiation, and it is roughly reciprocal to the signal-to-noise ratio (SNR). Therefore, $D^{*}$ is a good measure to evaluate the sensitivity of a detector normalized by the detector area $A_{D}$. If infrared light having stable and steady power is available for the study, a detector with a higher $D^{*}$ would have a higher throughput, since the apparatus noise is approximately constant.

The sensitivity of an MCT detector is known to depend on modulation frequency of an FT-IR, which has conventionally been discussed by using the parameter, D*.2 According to a 
former report, ${ }^{12} \mathrm{D}^{*}$ goes up rapidly when the modulation frequency is increased up to $1 \mathrm{kHz}$, while it exhibits a gradual increase in the range of $1 \mathrm{kHz}-1 \mathrm{MHz}$, which is, in fact, found in Figure 1 in the range of $20-150 \mathrm{kHz}$ by about only $5 \%$. Since thin film analysis requires high throughput measurements, the observed results indicate that the MCT detector should be used at a high modulation frequency as possible.

The use of an MCT detector at a high modulation frequency has another benefit that the collection time of spectra becomes shorter, which enables us to accumulate more scans in the same period of time to attain a better SNR. For example, multiple-angle incidence resolution spectrometry (MAIRS) that is powerful for thin film analysis ${ }^{8-10}$ requires repeated measurements at several angles of incidence, which spends much time for the measurements.

Then, measurement time of 2000 scans against the modulation frequency was investigated for both spectrometers as plotted in Figure 2. The number of 2000 scans is often employed in our laboratory to obtain spectra of ultrathin films with a good SNR. The results of the two spectrometers are close to each other particularly for a high modulation frequency.

When the moving mirror of Michelson's interferometer is moved at a constant rate of $\mathrm{v}$ in a period of time, $t$, the total running length of the moving mirror, $l$, is:

$$
\mathrm{vt}=\mathrm{I}
$$

Since the modulation frequency, $\mathrm{f}$, is related by Eq. 1 to the mirror velocity, $\mathrm{v}$, at the laser 
frequency, $\tilde{v}$, the product of $f$ and $t$ becomes a constant as formulated in Eq. 5.

$$
\mathrm{ft}=2 \tilde{v}
$$

In fact, the plot in Figure 2 roughly obeys the inverse proportional relationship. On closer inspection, however, the change in the measurement time becomes little especially above 100 $\mathrm{kHz}$, which is apparently out of Eq. 5. To make the results easier to understand, the same results are re-plotted in Figure 3, in which the measurement time, $t$, is plotted against the reciprocal mirror velocity, $1 / \mathrm{v}$.

The open and closed circles are roughly on the dashed and solid lines that are formulated as:

$$
(t-a)=\frac{l}{v}
$$

where $a$ is the intercept on the ordinate axis. Therefore, the slope of the line corresponds to the total running length of the mirror, $l$, and $a$ should correspond to the dead time wasted for the smooth mechanical movement of the moving mirror. Judging from Figure 3, $l$ and $a$ are calculated to be $0.516 \mathrm{~cm}$ and $0.338 \mathrm{sec}$ for the old spectrometer, whereas they are obtained to be $0.566 \mathrm{~cm}$ and $0.270 \mathrm{sec}$ for the new spectrometer, respectively, per scan. No significant difference has been found between the two spectrometers.

The resolution, $\Delta \tilde{v}$, is simply related to the maximum retardation of the moving mirror, $\Delta_{\max }:^{2}$ 


$$
(\Delta \tilde{v})=\left(\Delta_{\max }\right)^{-1}
$$

Since the resolution in the present study is $4 \mathrm{~cm}^{-1}, \Delta_{\max }$ is calculated to be $0.25 \mathrm{~cm}$ for a double-sided interferogram. Therefore, the observed running distances $(0.516$ and $0.566 \mathrm{~cm})$ are considered to involve much extra-length (about $0.30 \mathrm{~cm}$ ) for attaining a mechanical stability. The phenomenon of this time loss of the duty cycles is well known, and the analysis using the reciprocal velocity plot is found to be useful to determine an optimal fastest rapid scan rate.

Of note here is that the observed plots are out of the straight line for both spectrometers when the reciprocal of mirror velocity is less than $0.52 \mathrm{sec} \mathrm{cm}^{-1}$ that corresponds to the mirror velocity of $1.8988 \mathrm{~cm} \mathrm{sec}^{-1}$ or higher (the modulation frequency is $60 \mathrm{kHz}$ or higher). The plot therefore suggests that if the modulation frequency is increased more than $60 \mathrm{kHz}$, dead time would also be increased, which means that the efficiency of accumulation would be degraded.

Another issue has come up when absorbance spectra of a thin film were measured with a rapid scan. To investigate the measurement stability of an 'absorbance spectrum' of a very thin film, a practical sample of a five-monolayer LB film of cadmium stearate was measured with various modulation frequencies. The background spectra were collected by the use of a germanium substrate with no film. Representative five transmission spectra are presented in Figure 4, in which a whole wavenumber range of spectra measured with an MCT detector is shown. In the $\mathrm{C}-\mathrm{H}$ stretching vibration region, the anti-symmetric and symmetric $\mathrm{CH}_{2}$ stretching 
vibration bands appear strongly at 2917 and $2850 \mathrm{~cm}^{-1}$, respectively, because of the surface selection rule of the transmission spectrometry for an thin film with a molecular orientation of perpendicular stance. ${ }^{7}$ In the same manner, the anti-symmetric $\mathrm{COO}^{-}$stretching vibration $\left(v_{\mathrm{a}}\left(\mathrm{COO}^{-}\right)\right)$band appears strongly at $1540 \mathrm{~cm}^{-1}$. The most significant characteristic of the spectra is that the spectrum measured with a modulation frequency of $200 \mathrm{kHz}$ is largely influenced by fringes.

When a low wavenumber region is magnified as in Figure 5, the intensity of the $v_{a}\left(\mathrm{COO}^{-}\right)$ band is found to be quantitatively influenced by the fringe. In this figure, the five spectra are overlaid with each other, so that the peak tops of the $v_{a}\left(\mathrm{COO}^{-}\right)$band match each other. Only the spectrum with the modulation frequency of $200 \mathrm{kHz}$ has a shifted baseline to a higher value than those of the rest spectra. As a result, the band intensity is made decreased by about $5 \%$ when 200 $\mathrm{kHz}$ is employed. The reason the fringes appear is not clear, but it may be caused by mechanical instability with the very high scan rate irrespective of the sampling frequency considering the Nyquist frequency. In this manner, the highest modulation frequency is not suitable for measurements of spectra having minute absorbance, although it is suitable for time resolved measurements of spectra of quick phenomena.

Conclusion: Optimization of modulation frequency has proved to be necessary for better reliable measurements of spectra for very thin films. In the present case of the old and new spectrometers, 
the modulation frequency of $60 \mathrm{kHz}$ has been found most useful in terms of both high throughput and efficient accumulation, although the spectrometers have different sampling frequencies. In addition, instability of single-beam measurements with a too high modulation frequency was revealed by finding fringes on an absorbance spectrum. Even for using a time-consuming technique such as MAIRS ${ }^{10-12}$ for molecular orientation analysis in a thin film, a higher frequency near $100 \mathrm{kHz}$ would be employed as the highest possible. With the rapid scan condition, $163 \mathrm{sec}$ can be saved for 2000 scans, which saves 16 min more for a MAIRS analysis using six angles of incidence. A higher frequency than $100 \mathrm{kHz}$ would not work well because of the dead time on scanning.

Although the matter of unpleasant long scanning time can be overcome by reducing settle time, in the present paper, characteristics of FT-IR measurements for thin film analysis with respect to scanning rate have been discussed at the default settle time.

\section{Acknowledgement:}

The authors greatly thank Mr. Tatsuhiko Nakano and Shin-ichiro Hayashi, Thermo Fisher Scientific (Yokohama, Japan), for their very kind discussion and comments. They also thank a reviewer of this paper, who gave us very helpful comments to appropriately discuss the results.

This work was financially supported by Precursory Research for Embryonic Science and Technology (PRESTO), Japan Science and Technology Agency (T.H.), to whom the authors' 
thanks are due.

\section{References:}

[1] T. Hasegawa, Veeranjaneyulu Konka and Roger M. Leblanc, "FT-IR spectroscopy of ultrathin materials" in "Vibrational spectroscopy of biological and polymeric materials" V.G. Gregoriou and M.S. Braiman Ed. (Taylor \& Francis, Boca Raton) 2006.

[2] “Fourier Transform Infrared Spectrometry, $2^{\text {nd }}$ ed.” P.R. Griffiths and J.A. de Haseth Ed. (Wiley, Hoboken) 2007.

[3] T. Hasegawa, S. Takeda, A. Kawaguchi and J. Umemura, Langmuir 11 (1995) 1236.

[4] T. Hasegawa, J. Umemura and T. Takenaka, Thin Solid Films 210/211 (1992) 583.

[5] S.A. Francis and A.H. Ellison, J. Opt. Soc. Am. 49 (1959) 131.

[6] R.J. Greenler, J. Chem. Phys. 44 (1966) 310.

[7] J. Umemura, "Reflection-Absorption Spectroscopy of Thin Films on Metallic Substrates" in “Handbook of Vibrational Spectroscopy Vol. 2” J.M. Chalmers and P.R. Griffiths Ed. (Wiley, Chichester) 2002.

[8] T. Hasegawa, J. Phys. Chem. B 106 (2002) 4112.

[9] T. Hasegawa, Anal. Bioanal. Chem. 388 (2007) 7.

[10] T. Hasegawa, Appl. Spectrosc. Rev. 43 (2008) 181. 
[11] “Fourier Transform Infrared Spectrometry, $1^{\text {st }}$ ed.” P.R. Griffiths and J.A. de Haseth Ed. (Wiley, New York) 1986.

[12] P.R. Griffiths, J.A. de Haseth and L. V. Azarraga, Anal. Chem. 55 (1983) 1361A. 


\section{Figure captions:}

Figure 1: Intensity variation of single-beam spectra of air measured at $2600 \mathrm{~cm}^{-1}$ with an increase of modulation frequency.

Figure 2: Measurement time needed for 2000 scans at various modulation frequencies, a part of which is magnified and presented in the inset.

Figure 3: The measurement time data in Figure 2 are re-plotted against the reciprocal of mirror velocity. The theoretically explainable part is represented by the dashed line.

Figure 4: Representative five transmission absorbance spectra of a five-monolayer LB film of cadmium stearate on a germanium substrate. For an easy comparison, the base lines of the spectra are shifted.

Figure 5: A part of the spectra in Figure 4 is re-plotted. In this figure, the peak tops of the $v_{\mathrm{a}}\left(\mathrm{COO}^{-}\right)$band are matched, so that the baseline shift would clearly be recognized. 


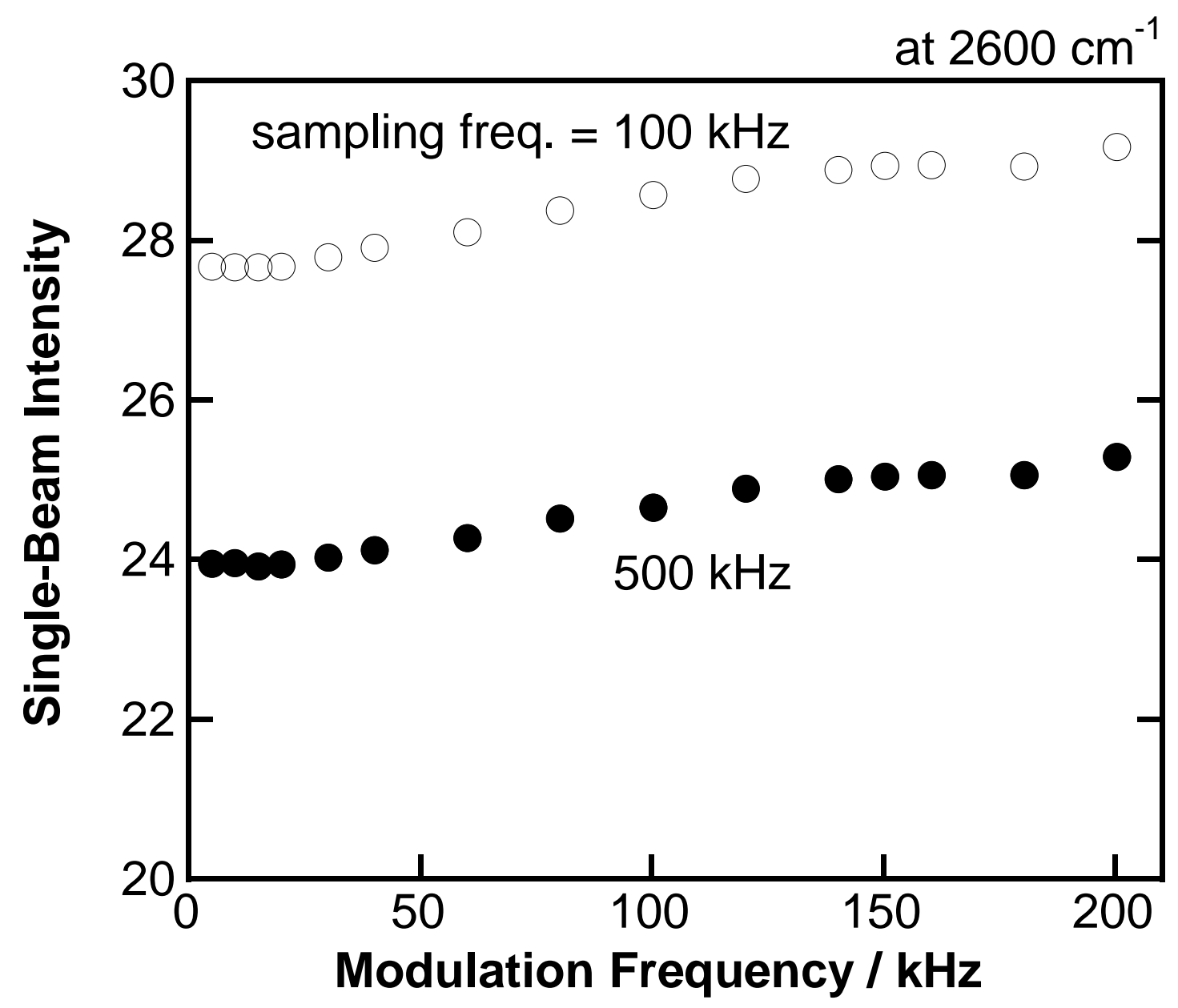

Figure 1 T. Hasegawa et al. 


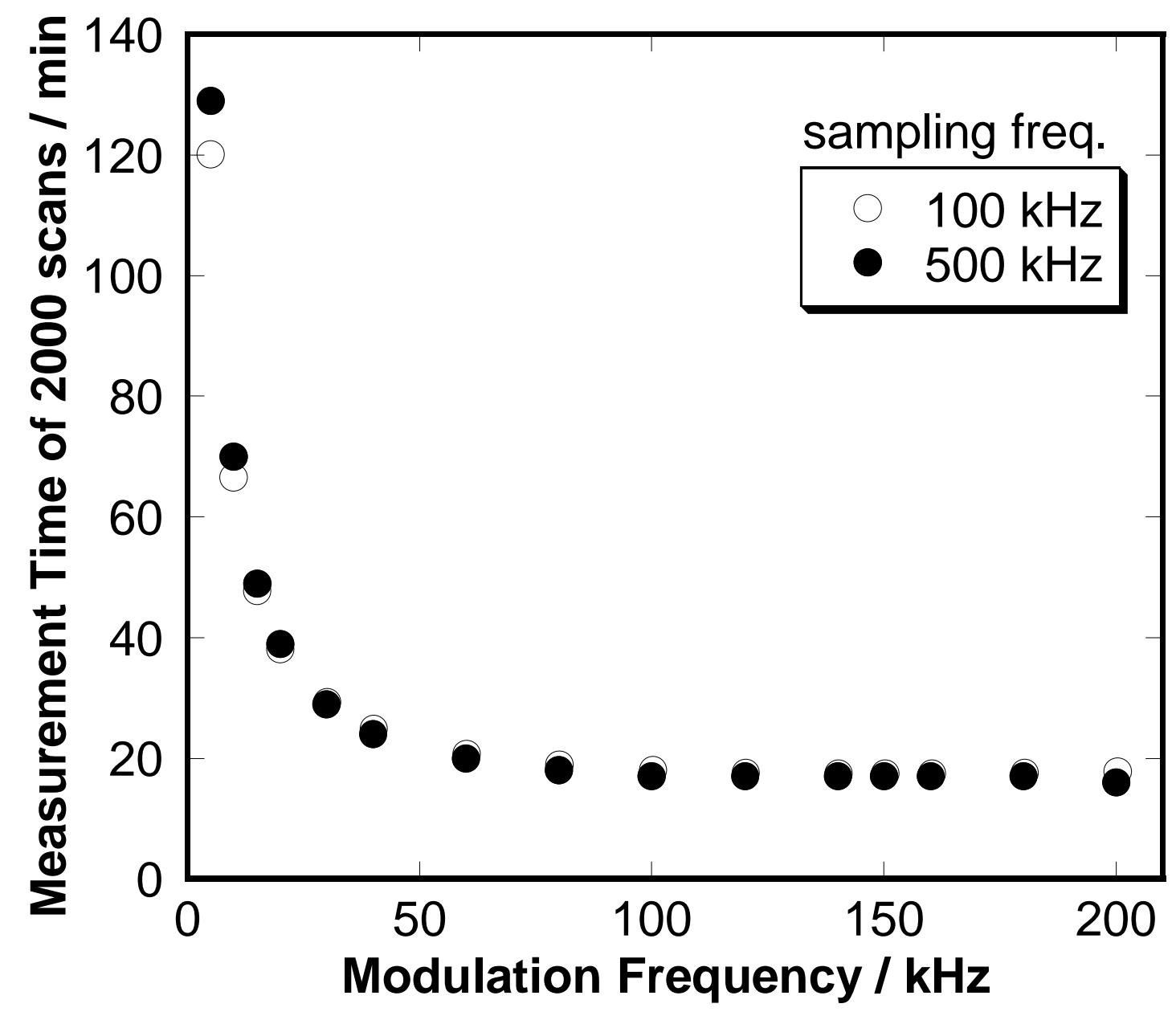

Figure 2 T. Hasegawa et al. 


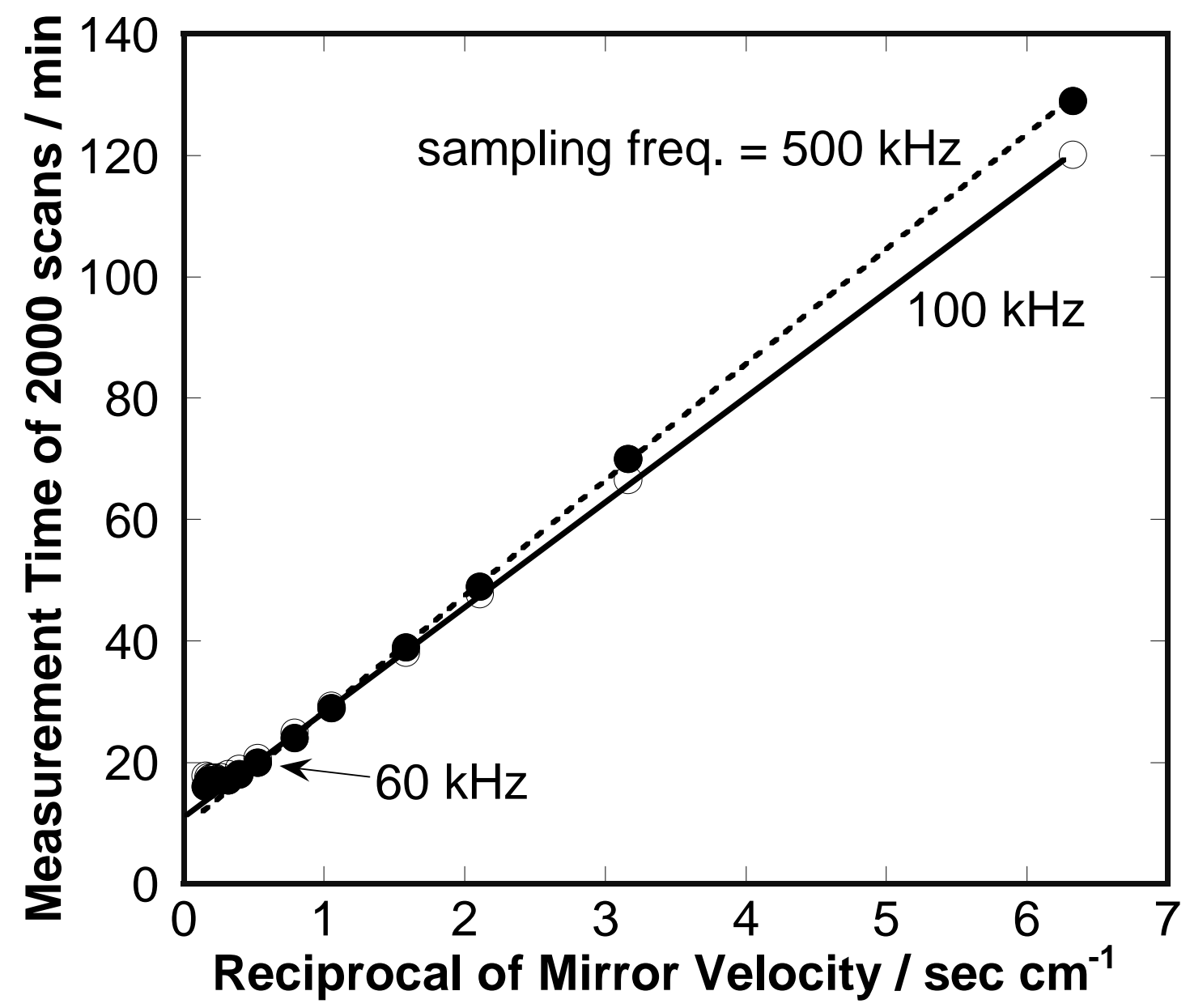

Figure 3 T. Hasegawa et al. 


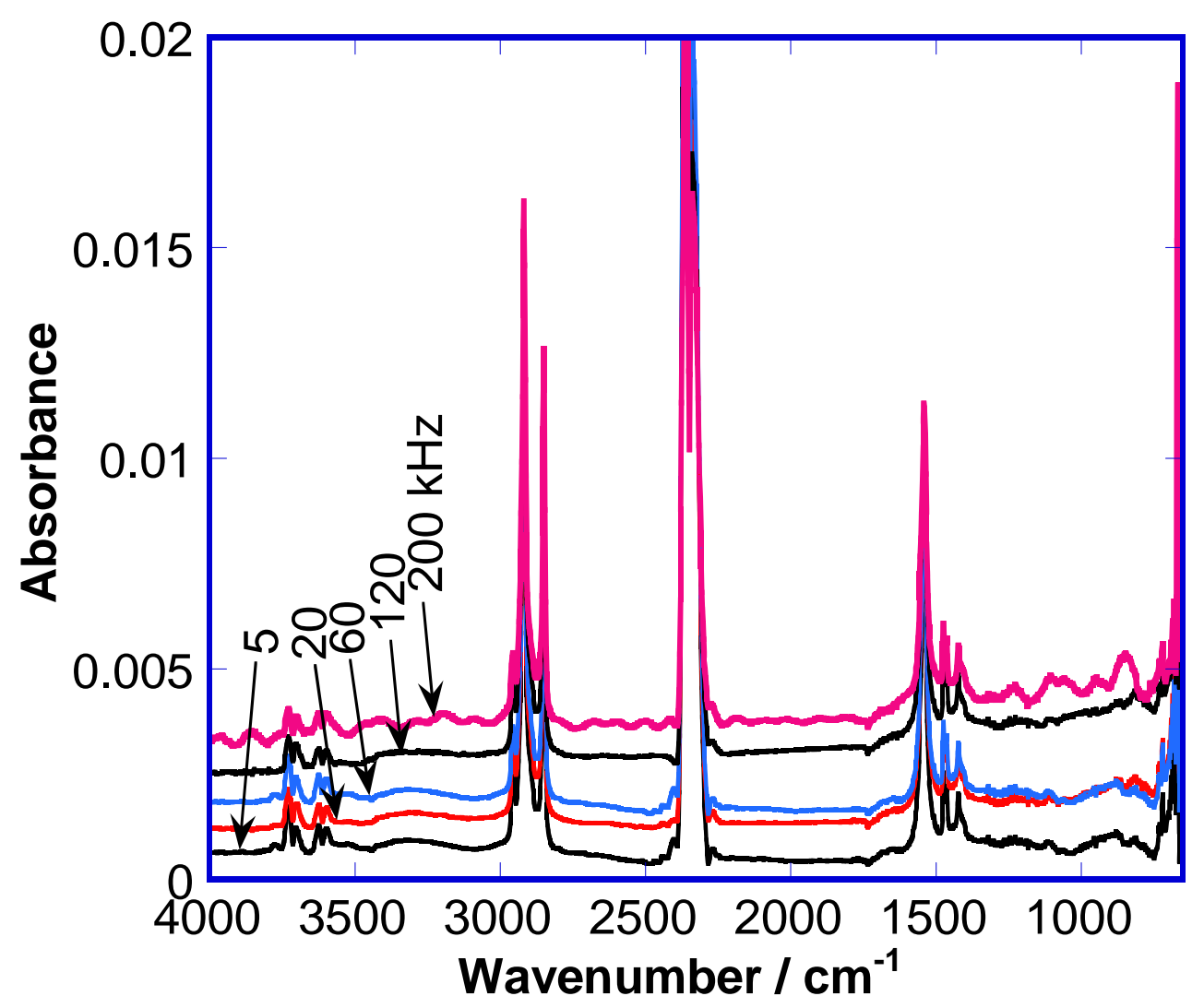

Figure 4 T. Hasegawa et al. 


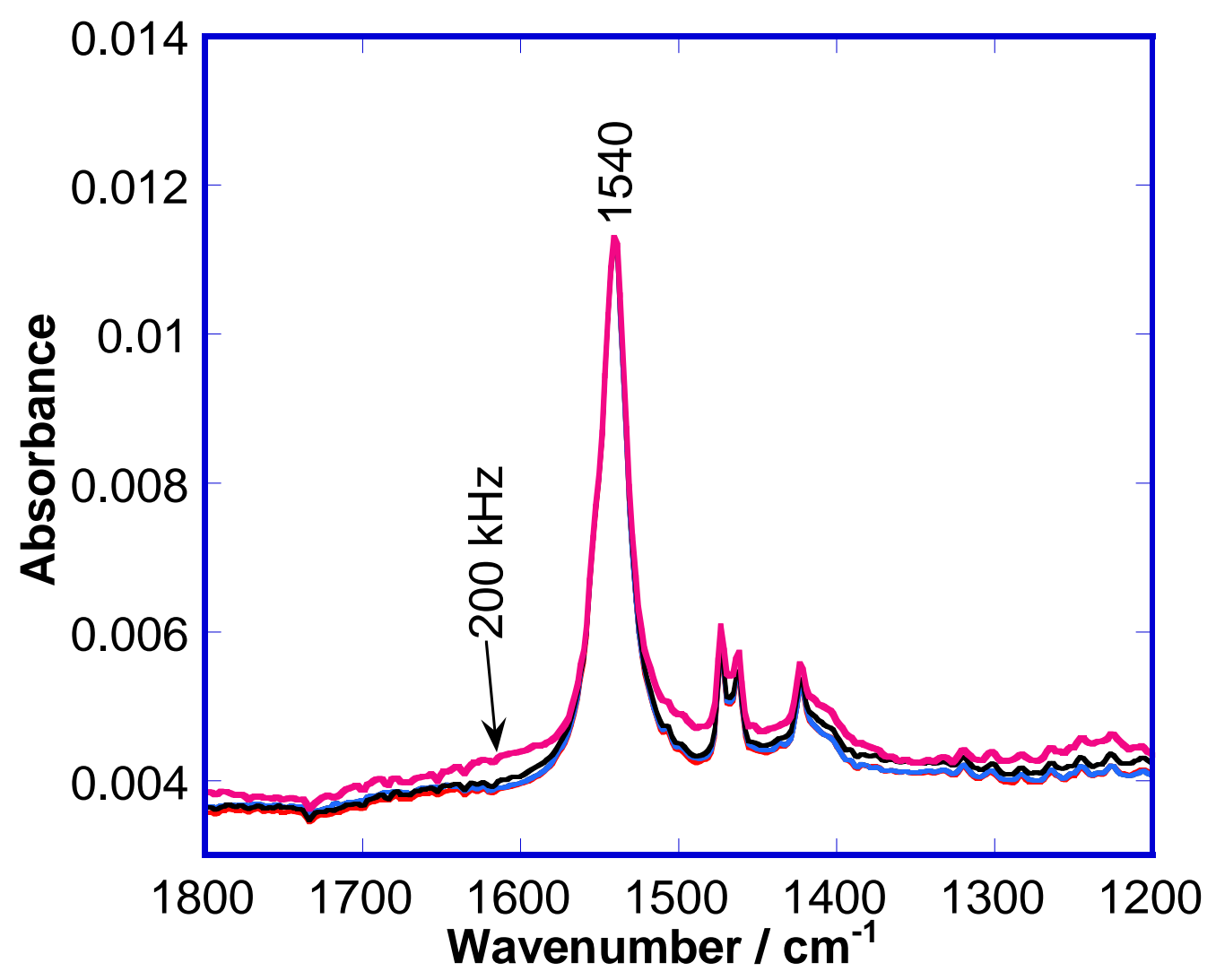

Figure 5 T. Hasegawa et al. 